\title{
Invariant Variation Problems
}

\author{
Emmy Noether
}

M. A. Tavel's English translation of "Invariante Variationsprobleme," Nachr. d. König. Gesellsch. d. Wiss. zu Göttingen, Math-phys. Klasse, 235-257 (1918), which originally appeared in Transport Theory and Statistical Physics, 1 (3), 183-207 (1971) 0

\begin{abstract}
The problems in variation here concerned are such as to admit a continuous group (in Lie's sense); the conclusions that emerge from the corresponding differential equations find their most general expression in the theorems formulated in Section 1 and proved in following sections. Concerning these differential equations that arise from problems of variation, far more precise statements can be made than about arbitrary differential equations admitting of a group, which are the subject of Lie's researches. What is to follow, therefore, represents a combination of the methods of the formal calculus of variations with those of Lie's group theory. For special groups and problems in variation, this combination of methods is not new; I may cite Hamel and Herglotz for special finite groups, Lorentz and his pupils (for instance Fokker), Weyl and Klein for special infinite groups 1 Especially Klein's second Note and the present developments have been mutually influenced by each other, in which regard I may refer to the concluding remarks of Klein's Note.
\end{abstract}

\section{$\S$ 1. Preliminary Remarks and Formulation of Theorems}

All functions occurring in the sequel are to be assumed analytic, or at least continuous and continuously differentiable a definite number of times, and unique in the interval considered.

By a "group of transformation," familiarly, is meant a system of transformations such that for each transformation, there exists an inverse contained in the system, and such that the composition of any two transformations of the system in turn belongs to the system. The group will be called a finite continuous group $\mathfrak{G}_{\rho}$ if its transformations are contained in a most general (transformation) depending analytically on $\rho$ essential parameters $\epsilon$ (i.e., the $\rho$ parameters are not to be representable as $\rho$ function of fewer parameters). Correspondingly, an infinite continuous group $\mathfrak{G}_{\infty \rho}$ is understood to be a group whose most general transformations depend on $\rho$ essential arbitrary functions $p(x)$ and their derivatives analytically, or at least in a continuous and finite-fold continuously differentiable manner. The group depending on infinitely many parameters but not on arbitrary functions stands as an intermediate term between the two. Finally, a group depending both on arbitrary functions and on parameters is called a mixed group 2

Let $x_{1}, \ldots, x_{n}$ be independent variables and $u_{1}(x), \ldots, u_{\mu}(x)$ functions depending upon them. If the $x$ 's and $u$ 's are subjected to the transformations of a group, then, by hypothesis of invertibility

\footnotetext{
${ }^{0}$ This paper is reproduced by Frank Y. Wang (fwang@lagcc.cuny.edu) with LATEX.

${ }^{1}$ Hamel, Math. Ann. 59 and Z. f. Math. u. Phys. 50. Herglotz, Ann. d. Phys. (4) 36, esp. § 9, p. 511. Fokker, Verslag d. Amsterdamer Akad. Jan. 27, 1917. For further bibliography, compare Klein's second Note, Göttinger Nachrichten, July 19, 1918. The recently published work by Kneser (Math. Zschr. 2) deals with the setting up of invariants by a similar method.

${ }^{2}$ Lie, in "Grundlagen für die Theorie der Unendlichen kontinuierlichen Transformationsgruppen" (Foundations of the theory of infinite continuous groups of transformations), Ber. d. K. Sachs. Ges. d. Wissensch 1981 (cited as Grundlagen), defines the infinite continuous group as a group of transformations which are given by the most general solutions of a system of partial differential equations, provided these solutions do not depend only on a finite number of parameters. One of the above-mentioned types differing from the finite group will thus be thereby obtained; whereas conversely the limiting case of infinitely many parameters need not necessarily satisfy a system of differential equations.
} 
of the transformations, there must again be exactly $n$ independent variables $y_{1}, \ldots, y_{n}$ among the transformed quantities; let the others depending upon them be designated by $v_{1}(y), \ldots, v_{\mu}(y)$. In the transformations, the derivatives of the $u$ 's with respect to the $x$ 's, namely $\frac{\partial u}{\partial x}, \frac{\partial^{2} u}{\partial x^{2}}, \ldots$ may also occur 3 A function is called an invariant of the group if there subsists a relationship

$$
P\left(x, u, \frac{\partial u}{\partial x}, \frac{\partial^{2} u}{\partial x^{2}}, \ldots\right)=P\left(y, v, \frac{\partial v}{\partial y}, \frac{\partial^{2} v}{\partial y^{2}}, \ldots\right) .
$$

In particular, then, an integral $I$ will be an invariant of the group if there subsists a relationship

$$
I=\int \ldots \int f\left(x, u, \frac{\partial u}{\partial x}, \frac{\partial^{2} u}{\partial x^{2}} \ldots\right) d x=\int \ldots \int f\left(y, v, \frac{\partial v}{\partial y}, \frac{\partial^{2} v}{\partial y^{2}} \ldots\right) d y \quad \text { 幽 }
$$

integrated over an arbitrary real $x$-interval and the corresponding $y$-interval 5

Then, for an arbitrary, not necessarily invariant integral $I$, I form the first variation $\delta I$ and transform it by partial integration according to the rules of the calculus of variations. As we know, provided $\delta u$ with all derivatives that occur is assumed to vanish at the boundary, while otherwise arbitrary,

$$
\delta I=\int \ldots \int \delta f d x=\int \ldots \int\left(\sum \psi_{i}\left(x, u, \frac{\partial u}{\partial x}, \ldots\right) \delta u_{i}\right) d x
$$

where $\psi$ stands for the Lagrange expressions, i.e., the left-hand sides of the Lagrange equations of the corresponding variation problem $\delta I=0$. To this integral relationship there corresponds an integral-free identity in $\delta u$ and its derivatives, generated by writing in the boundary terms as well. As the partial integration shows, these boundary terms are integrals over divergences, i.e., over expressions

$$
\operatorname{Div} A=\frac{\partial A_{1}}{\partial x_{1}}+\ldots+\frac{\partial A_{n}}{\partial x_{n}}
$$

where $A$ is linear in $\delta u$ and its derivatives. Hence

$$
\sum \psi_{i} \delta u_{i}=\delta f+\operatorname{Div} A
$$

If in particular $f$ contains only first derivatives of the $u$ 's, then in the case of the single integral the identity (3) is identical with what Heun calls the "central equation of Lagrange"

$$
\sum \psi_{i} \delta u_{i}=\delta f-\frac{d}{d x}\left(\sum \frac{\partial f}{\partial u_{i}^{\prime}} \delta u_{i}\right), \quad\left(u_{i}^{\prime}=\frac{d u_{i}}{d x}\right)
$$

whereas for the $n$-fold integral, (3) goes over into

$$
\sum \psi_{i} \delta u_{i}=\delta f-\frac{\partial}{\partial x_{1}}\left(\sum \frac{\partial f}{\partial \frac{\partial u_{i}}{\partial x_{1}}} \delta u_{i}\right)-\ldots-\frac{\partial}{\partial x_{n}}\left(\sum \frac{\partial f}{\partial \frac{\partial u_{i}}{\partial x_{n}}} \delta u_{i}\right) .
$$

\footnotetext{
${ }^{3}$ I suppress the subscripts, insofar as feasible, even in summations; thus, $\frac{\partial^{2} u}{\partial x^{2}}$ for $\frac{\partial^{2} u_{\alpha}}{\partial x_{\beta} \partial x_{\gamma}}$, etc.

${ }^{4}$ By way of abbreviation, I write $d x, d y$ for $d x_{1} \ldots d x_{n}, d y_{1} \ldots d y_{n}$.

${ }^{5}$ All arguments $x, u, \epsilon, p(x)$ occurring in the transformations are to be assumed real, whereas the coefficients may be complex. But since the final results are concerned with identities in the $x$ 's, $u$ 's, parameters and arbitrary functions, these hold also for complex values, provided only that all functions that occur are assumed analytic. A large portion of the results, incidentally, can be justified without integrals, so that here the restriction to reals is not necessary even to the arguments. On the other hand, the developments at the close of Section 2 and beginning of Section 5 do not appear to be feasible without integrals.
} 
For the single integral and $\kappa$ derivatives of the $u$ 's, (3) is given by

$$
\begin{aligned}
\sum \psi_{i} \delta u_{i} & =\delta f- \\
- & \frac{d}{d x}\left\{\sum\left(\left(\begin{array}{l}
1 \\
1
\end{array}\right) \frac{\partial f}{\partial u_{i}^{(1)}} \delta u_{i}+\left(\begin{array}{l}
2 \\
1
\end{array}\right) \frac{\partial f}{\partial u_{i}^{(2)}} \delta u_{i}^{(1)}+\ldots+\left(\begin{array}{c}
\kappa \\
1
\end{array}\right) \frac{\partial f}{\partial u_{i}^{(\kappa)}} \delta u_{i}^{(\kappa-1)}\right)\right\}+ \\
+ & \frac{d^{2}}{d x^{2}}\left\{\sum\left(\left(\begin{array}{l}
2 \\
2
\end{array}\right) \frac{\partial f}{\partial u_{i}^{(2)}} \delta u_{i}+\left(\begin{array}{l}
3 \\
2
\end{array}\right) \frac{\partial f}{\partial u_{i}^{(3)}} \delta u_{i}^{(1)}+\ldots+\left(\begin{array}{c}
\kappa \\
2
\end{array}\right) \frac{\partial f}{\partial u_{i}^{(\kappa)}} \delta u_{i}^{(\kappa-2)}\right)\right\}+\ldots \\
& +(-1)^{\kappa} \frac{d^{\kappa}}{d x^{\kappa}}\left\{\sum\left(\begin{array}{l}
\kappa \\
\kappa
\end{array}\right) \frac{\partial f}{\partial u_{i}^{(\kappa)}} \delta u_{i}\right\}
\end{aligned}
$$

and a corresponding identity holds for the $n$-fold integral; in particular, $A$ contains $\delta u$ as far as the $(\kappa-1)$ st derivative. The fact that (4), (5), and (6) actually define the Lagrange expressions $\psi_{i}$ follows from the fact that the combinations of the right-hand sides eliminate all higher derivatives of the $\delta u$ 's, while on the other hand the relation (2), to which the partial integration leads uniquely, is satisfied.

Now in the following we shall be concerned with these two theorems:

I. If the integral $I$ is invariant with respect to a $\mathfrak{G}_{\rho}$, then $\rho$ linearly independent combinations of the Lagrange expressions become divergences - and from this, conversely, invariance of $I$ with respect to a $\mathfrak{G}_{\rho}$ will follow. The theorem holds good even in the limiting case of infinitely many parameters.

II. If the integral $I$ is invariant with respect to a $\mathfrak{G}_{\infty \rho}$ in which the arbitrary functions occur up to the $\sigma$-th derivative, then there subsist $\rho$ identity relationships between the Lagrange expressions and their derivatives up to the $\sigma$-th order. In this case also, the converse holds 6

For mixed groups, the statements of both theorems hold; that is, both dependencies and divergence relations independent thereof occur.

Passing over from these identities to the corresponding variation problem, i.e., putting $\psi=07$ Theorem I in the one-dimensional case - where the divergence goes over into a total differential - asserts the existence of $\rho$ first integrals, between which, however, non-linear dependencies may subsist 8 in the multidimensional case, the divergence equations often referred to of late as "laws of conservation" are obtained; Theorem II states that $\rho$ of the Lagrange equations are a consequence of the rest.

The simplest example of Theorem II - without converse - is afforded by the Weierstrass parametric representation; here the integral, with homogeneity of first order, is as we know invariant if the independent variable $x$ is replaced by an arbitrary function of $x$ that leaves $u$ unchanged $(y=p(x)$; $v_{i}(y)=u_{i}(x)$ ). Thus one arbitrary function occurs, but without derivatives, and to this corresponds the known linear relationship among the Lagrange expressions themselves $\sum \psi_{i} \frac{d u_{i}}{d x}=0$. Another example is presented by the "general theory of relativity" of the physicists; there we have the group of all transformations $y_{i}=p_{i}(x)$ of the $x$ 's, while the $u$ 's (designated as $g_{\mu \nu}$ and $q$ ) are subjected to the transformations thereby induced for the coefficients of a quadratic and linear differential form transformations which contain the first derivatives of the arbitrary function $p(x)$. To this correspond the familiar $n$ dependencies between the Lagrange expressions and their first derivatives 9

If in particular we specialize the group by allowing no derivatives of the $u(x)$ 's in the transformations, and moreover let the transformed independent quantities depend only on the $x$ 's, not on the $u$ 's, then (as is shown in Section 5) the invariance of $I$ entails the relative invariance of $\sum \psi_{i} \delta u_{i}[0$

\footnotetext{
${ }^{6}$ For certain trivial exceptions, compare Section 2, Note 13.

${ }^{7}$ Somewhat more generally, we may alternatively put $\psi_{i}=T_{i}$; cf. Section 3, Note 15.

${ }^{8} \mathrm{Cf}$. close of Section 3.

${ }^{9}$ Cf. e.g., Klein's presentation.

${ }^{10}$ That is, $\sum \psi_{i} \delta u_{i}$ acquires a factor upon transformation.
} 
and likewise of the divergences occurring in Theorem I, once the parameters are subjected to suitable transformations. For Theorem II, similarly, we get relative invariance of the left-hand sides of the dependencies as associated with the aid of the arbitrary functions; and as a consequence of this, another function whose divergence vanishes identically and admits of the group - mediating, in the physicists' theory of relativity, the connection between dependencies and the law of conservation of energy 11 Theorem II, finally, in terms of group theory, furnishes the proof of a related Hilbertian assertion about the failure of laws of conservation of energy proper in "general relativity." With these supplementary remarks, Theorem I comprises all theorems on first integrals known to mechanics etc., while Theorem II may be described as the utmost possible generalization of the "general theory of relativity" in group theory.

\section{$\S 2$. Divergence Relationships and Dependencies}

Let $\mathfrak{G}$ be a - finite or infinite - continuous group; then it is always possible to arrange for the zero values of the parameters $\epsilon$, or of the arbitrary function $p(x)$, to correspond to the identity transformation 12 The most general transformation will therefore be of the form

$$
\begin{gathered}
y_{i}=A_{i}\left(x, u, \frac{\partial u}{\partial x}, \ldots\right)=x_{i}+\Delta x_{i}+\ldots \\
v_{i}(y)=B_{i}\left(x, u, \frac{\partial u}{\partial x}, \ldots\right)=u_{i}+\Delta u_{i}+\ldots
\end{gathered}
$$

where $\Delta x_{i}, \Delta u_{i}$ stand for the terms of lowest dimension in $\epsilon$, or $p(x)$ and its derivatives; in which, in fact, they will be assumed linear. As will afterwards appear, this is no restriction of generality.

Now let the integral $I$ be an invariant with respect to $\mathfrak{G}$, satisfying, that is, the relationship (1). Then in particular, $I$ will also be invariant with respect to the infinitesimal transformation

$$
y_{i}=x_{i}+\Delta x_{i} ; \quad v_{i}(y)=u_{i}+\Delta u_{i} ;
$$

contained in $\mathfrak{G}$, and for this relation (1) goes over into

$$
0=\Delta I=\int \ldots \int f\left(y, v(y), \frac{\partial v}{\partial y}, \ldots\right) d y-\int \ldots \int f\left(x, u(x), \frac{\partial u}{\partial x}, \ldots\right) d x,
$$

where the first integral is to be extended over the $x+\Delta x$ interval corresponding to the $x$-interval. But this integration may alternatively be transformed into an integration over the $x$-interval, by virtue of the transformation, valid for infinitesimal $\Delta x$,

$$
\int \ldots \int f\left(y, v(y), \frac{\partial v}{\partial y}, \ldots\right) d y=\int \ldots \int f\left(x, v(x), \frac{\partial v}{\partial x}, \ldots\right) d x+\int \ldots \int \operatorname{Div}(f \cdot \Delta x) d x .
$$

So if in place of the infinitesimal transformation $\Delta u$, we introduce the variation

$$
\bar{\delta} u_{i}=v_{i}(x)-u_{i}(x)=\Delta u_{i}-\sum \frac{\partial u_{i}}{\partial x_{\lambda}} \Delta x_{\lambda},
$$

then (7) and (8) go over into

$$
0=\int \ldots \int\{\bar{\delta} f+\operatorname{Div}(f \cdot \Delta x)\} d x
$$

The right-hand side is the familiar formula for simultaneous variation of the dependent and independent variables. Since the relation (10) is satisfied for integration over any arbitrary interval, the

\footnotetext{
${ }^{11}$ Compare Klein's second note.

${ }^{12}$ Cf. e.g., Lie, Grundlagen, p. 331. Where arbitrary functions are concerned, the special values $a^{\sigma}$ of the parameters are to be replaced by fixed functions $p^{\sigma}, \frac{\partial p^{\sigma}}{\partial x}, \ldots ;$ and correspondingly, the values $a^{\sigma}+\epsilon$ by $p+p(x), \frac{\partial p^{\sigma}}{\partial x}+\frac{\partial p}{\partial x}$, etc.
} 
integrand must vanish identically; therefore Lie's differential equations for the invariance of $I$ goes over into the relation

$$
\bar{\delta} f+\operatorname{Div}(f \cdot \Delta x)=0 .
$$

If in this, by (3), $\bar{\delta} f$ is expressed in terms of the Lagrange expressions, we get

$$
\sum \psi_{i} \bar{\delta} u_{i}=\operatorname{Div} B \quad(B=A-f \cdot \Delta x)
$$

and this relationship, therefore, for every invariant integral $I$, represents an identity in all arguments that occur; it is the required form of Lie's differential equations for $I 13$

Now for the present let $\mathfrak{G}$ be taken to be a finite continuous group $\mathfrak{G}_{\rho}$; since by hypothesis $\Delta u$ and $\Delta x$ are linear in the parameters $\epsilon_{1}, \ldots, \epsilon_{\rho}$, hence by (9) the same holds for $\bar{\delta} u$ and its derivatives; therefore $A$ and $B$ are linear in the $\epsilon$ 's. So if I let

$$
B=B^{(1)} \epsilon_{1}+\ldots+B^{(\rho)} \epsilon_{\rho} ; \quad \bar{\delta} u=\bar{\delta} u^{(1)} \epsilon_{1}+\ldots+\bar{\delta} u^{(\rho)} \epsilon_{\rho},
$$

where, that is $\bar{\delta} u^{(1)}, \ldots$ are functions of $x, u, \frac{\partial u}{\partial x}, \ldots$, the required divergence relationships follow from (12):

$$
\sum \psi_{i} \bar{\delta} u_{i}^{(1)}=\operatorname{Div} B^{(1)} ; \quad \ldots \quad \sum \psi_{i} \bar{\delta} u_{i}^{(\rho)}=\operatorname{Div} B^{(\rho)} .
$$

Thus $\rho$ linearly independent combinations of the Lagrange expressions become divergences; the linear independence follows from the fact that by (9), $\bar{\delta} u=0, \Delta x=0$ would entail $\Delta u=0, \Delta x=0$, or in other words a dependency between the infinitesimal transformations. But by hypothesis, none such is satisfied for any value of the parameters, since otherwise the $\mathfrak{G}_{\rho}$ regenerated by integration from the infinitesimal transformations would depend on fewer than $\rho$ essential parameters. But the further possibility $\bar{\delta} u=0, \operatorname{Div}(f \cdot \Delta x)=0$ was excluded. These conclusions hold good even in the limiting case of infinitely many parameters.

Now let $\mathfrak{G}$ be an infinite continuous group $\mathfrak{G}_{\infty}$; then $\bar{\delta} u$ and its derivatives, and hence $B$ also, will again be linear in the arbitrary functions of $p(x)$ and their derivatives 14 independently of (12), further, by substitution of the values of $\bar{\delta} u$, let

$$
\sum \psi_{i} \bar{\delta} u_{i}=\sum_{\lambda, i} \psi_{i}\left\{a_{i}^{(\lambda)}(x, u, \ldots) p^{(\lambda)}(x)+b_{i}^{(\lambda)}(x, u, \ldots) \frac{\partial p^{(\lambda)}}{\partial x}+\ldots+c_{i}^{(\lambda)}(x, u, \ldots) \frac{\partial^{\sigma} p^{(\lambda)}}{\partial x^{\sigma}}\right\} .
$$

Now, by the identity

$$
\varphi(x, u, \ldots) \frac{\partial^{\tau} p(x)}{\partial x^{\tau}}=(-1)^{\tau} \cdot \frac{\partial^{\tau} \varphi}{\partial x^{\tau}} \cdot p(x) \bmod \text { Divergences }
$$

and analogously to the partial integration formula, the derivatives of $p$ can be replaced by $p$ itself and by divergences that will be linear in $p$ and its derivatives; hence we get

$$
\sum \psi_{i} \bar{\delta} u_{i}=\sum_{\lambda}\left\{\left(a_{i}^{(\lambda)} \psi_{i}\right)-\frac{\partial}{\partial x}\left(b_{i}^{(\lambda)} \psi_{i}\right)+\ldots+(-1)^{\sigma} \frac{\partial^{\sigma}}{\partial x^{\sigma}}\left(c_{i}^{(\lambda)} \psi_{i}\right)\right\} p^{(\lambda)}+\operatorname{Div} \Gamma
$$

and in conjunction with (12)

$$
\sum\left\{\left(a_{i}^{(\lambda)} \psi_{i}\right)-\frac{\partial}{\partial x}\left(b_{i}^{(\lambda)} \psi_{i}\right)+\ldots+(-1)^{\sigma} \frac{\partial^{\sigma}}{\partial x^{\sigma}}\left(c_{i}^{(\lambda)} \psi_{i}\right)\right\} p^{(\lambda)}=\operatorname{Div}(B-\Gamma) .
$$

I now form the $n$-fold integral over (15), extended over any interval; and choose the $p(x)$ 's such that they, with all derivatives occurring in $(B-\Gamma)$, will vanish at the boundary. Since the integral

\footnotetext{
${ }^{13}(12)$ goes over into $0=0$ for the trivial case - which can occur only if $\Delta x, \Delta u$ depend also on derivatives of the $u$ 's - when $\operatorname{Div}(f \cdot \Delta x)=0, \bar{\delta} u=0$; thus these infinitesimal transformations are always to be eliminated from the groups, and only the number of remaining parameters, or arbitrary functions, is to be counted in the formulation of the theorems. Whether the remaining infinitesimal transformations still form a group must be left moot.

${ }^{14}$ That it signifies no restriction to assume the $p$ 's free from $u, \frac{\partial u}{\partial x}$, is shown by the converse.
} 
over a divergence reduces to a boundary integral, then, the integral over the left side of (15) will also vanish for $p(x)$ 's which are arbitrary except that they and sufficiently many of their derivatives vanish at the boundary; and thence follows, by known inferences, the vanishing of the integrand for every $p(x)$, or in other words the $\rho$ relationships:

$$
\sum\left\{\left(a_{i}^{(\lambda)} \psi_{i}\right)-\frac{\partial}{\partial x}\left(b_{i}^{(\lambda)} \psi_{i}\right)+\ldots+(-1)^{\sigma} \frac{\partial^{\sigma}}{\partial x^{\sigma}}\left(c_{i}^{(\lambda)} \psi_{i}\right)\right\}=0 \quad(\lambda=1,2, \ldots, \rho) .
$$

These are the required dependencies between the Lagrange expressions and their derivatives for invariance of $I$ with respect to $\mathfrak{G}_{\infty \rho}$; the linear independence is proved as above, since the converse leads back to (12), and since we can again argue back from the infinitesimal transformations to the finite ones, as will be explained more fully in Section 4. In the case of a $\mathfrak{G}_{\infty \rho}$, that is to say, even in the infinitesimal transformations there always occur $\rho$ arbitrary transformations. Equation (15) and (16) further entail $\operatorname{Div}(B-\Gamma)=0$.

If, as corresponds to a "mixed group," $\Delta x$ and $\Delta u$ are taken linear in the $\epsilon$ 's and $p(x)$ 's, then we see, by equating first the $p(x)$ 's and then the $\epsilon$ 's to zero, that both divergence relationships (13) and dependencies (16) hold.

\section{$\S 3$. Converse in Case of Finite Group}

To prove the converse, we are first to run through essentially the foregoing arguments in reverse order. From the fact of (13), by multiplication by the $\epsilon$ 's and adding, the fact of (12) follows; and thence, by virtue of the identity (3), a relationship $\bar{\delta} f+\operatorname{Div}(A-B)=0$. So if we put $\Delta x=\frac{1}{f}(A-B)$, we have thereby arrived at (11); whence finally, by integration, there follows (7), $\Delta I=0$, or in other words the invariance of $I$ with respect to the infinitesimal transformation determined by $\Delta x, \Delta u$, where $\Delta u$ 's by virtue of (9) are determined from $\Delta x$ and $\bar{\delta} u$, and $\Delta x$ and $\Delta u$ become linear in the parameters. But $\Delta I=0$ entails, in known manner, the invariance of $I$ with respect to the finite transformations generated by integration of the simultaneous system

$$
\frac{d x_{i}}{d t}=\Delta x_{i} ; \quad \frac{d u_{i}}{d t}=\Delta u_{i} ; \quad\left(x_{i}=y_{i}, u_{i}=v_{i}, \text { for } t=0\right) .
$$

These finite transformations contain $\rho$ parameters $a_{1} \ldots a_{\rho}$, namely the combinations $t \epsilon_{1}, \ldots, t \epsilon_{\rho}$. From the assumption that there are $\rho$ and only $\rho$ linearly independent divergence relationships (13), it follows further that the finite transformations, once they do not contain the derivatives $\frac{\partial u}{\partial x}$, always form a group. For in the contrary case, at least one infinitesimal transformation generated by Lie's bracketing process would fail to be a linear combination of the other $\rho$; and since $I$ admits of this transformation also, there would be more than $\rho$ linearly independent divergence relationships; or else that infinitesimal transformation would be of the special form where $\bar{\delta} u=0, \operatorname{Div}(f \cdot \Delta x)=0$, but in that case $\Delta x$ or $\Delta u$, contrary to hypothesis, would depend on derivatives. Whether this case can arise when derivatives occur in $\Delta x$ or $\Delta u$ must be left moot; in that case, the $\Delta x$ determined above must be augmented by all functions $\Delta x$ for which $\operatorname{Div}(f \cdot \Delta x)=0$ to restore the group property, but by agreement the parameters thereby adjoined are not to count. This completes the proof of the converse.

From this conversion, it follows further that $\Delta x$ and $\Delta u$ can actually be assumed linear in the parameters. For if $\Delta u$ and $\Delta x$ were forms of higher degree in $\epsilon$, then by the linear independence of the power products of the $\epsilon$ 's, quite analogous relations (13) would follow, only in greater number, from which, by the converse, invariance of $I$ follows with respect to a group whose infinitesimal transformations contain the parameters linearly. If this group is to contain exactly $\rho$ parameters, then linear dependencies must subsist between the divergence relationships originally obtained through the terms of higher degree in $\epsilon$.

Let us add the remark that in the case where $\Delta x$ and $\Delta u$ also contain derivatives of the $u$ 's, the finite transformations may depend on infinitely many derivatives of the $u$ 's; for in that case the 
integration of (17), in the determination of $\frac{d^{2} x_{i}}{d t^{2}}, \frac{d^{2} u_{i}}{d t^{2}}$ leads to $\Delta\left(\frac{\partial u}{\partial x_{\kappa}}\right)=\frac{\partial \Delta u}{\partial x_{\kappa}}-\sum_{\lambda} \frac{\partial u}{\partial x_{\lambda}} \frac{\partial \Delta x_{\lambda}}{\partial x_{\kappa}}$, so that the number of derivatives in general increases at each step. By way of example, say,

$$
\begin{gathered}
f=\frac{1}{2} u^{\prime 2} ; \quad \psi=-u^{\prime \prime} ; \quad \psi \cdot x=\frac{d}{d x}\left(u-u^{\prime} x\right) ; \quad \bar{\delta} u=x \cdot \epsilon ; \\
\Delta x=\frac{-2 u}{u^{\prime 2}} \epsilon ; \quad \Delta u=\left(x-\frac{2 u}{u^{\prime}}\right) \cdot \epsilon
\end{gathered}
$$

Since the Lagrange expressions of a divergence vanish identically, the converse shows, finally, the following: if $I$ admits of a $\mathfrak{G}_{\rho}$, then any integral that differs from $I$ only by a boundary integral, i.e., by an integral over a divergence, likewise admits of a $\mathfrak{G}_{\rho}$ having the same $\bar{\delta} u$ 's whose infinitesimal transformations will in general contain derivatives of the $u$ 's. Thus for instance, corresponding to the above example, $f^{*}=\frac{1}{2}\left\{u^{\prime 2}-\frac{d}{d x}\left(\frac{u^{2}}{x}\right)\right\}$ admits of the infinitesimal transformation $\Delta u=x \epsilon$, $\Delta x=0$; while derivatives of the $u$ 's occur in the infinitesimal transformations corresponding to $f$.

Passing over to the variations problem, i.e., putting $\psi_{i}=015$ (13) goes over into the equation Div $B^{(1)}=0, \ldots$, Div $B^{(\rho)}=0$, often referred to as "laws of conservation." In the one-dimensional

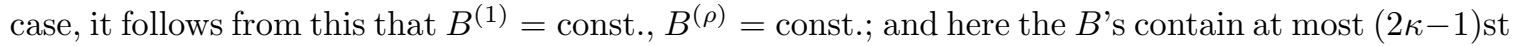
derivatives of the $u$ 's (by $(6)$ ), provided $\Delta u$ and $\Delta x$ contain no higher derivatives than the $\kappa$-th one occurring in $f$. Since $2 \kappa$-th derivatives in general occur in $\psi 16$ therefore, we have the existence of $\rho$ first integrals. That there may be non-linear dependencies among these is again shown by the above $f$. To the linearly independent $\Delta u=\epsilon_{1}, \Delta x=\epsilon_{2}$ there correspond the linearly independent relations $u^{\prime \prime}=\frac{d}{d x} u^{\prime} ; u^{\prime \prime} \cdot u^{\prime}=\frac{1}{2} \frac{d}{d x}\left(u^{\prime}\right)^{2}$; whereas between the first integrals $u^{\prime}=$ const., $u^{\prime 2}=$ const. a nonlinear dependency exists. This relates to the elementary case where $\Delta u, \Delta x$ contain no derivatives of the $u$ 's 17

\section{$\S$ 4. Converse in Case of Infinite Group}

First let us show that the assumption of linearity of $\Delta x$ and $\Delta u$ constitutes no restriction, a conclusion which follows, even without the converse, from the fact that $\mathfrak{G}_{\infty \rho}$ formally depends on $\rho$ and only $\rho$ arbitrary functions. For it turns out that in the non-linear case, upon composition of the transformations, whereby the terms of lowest order are added together, the number of arbitrary functions would increase. In fact, say, let

$$
\begin{aligned}
& y=A\left(x, u, \frac{\partial u}{\partial x}, \ldots ; p\right)=x+\sum a(x, u, \ldots) p^{\nu}+b(x, u, \ldots) p^{\nu-1} \frac{\partial p}{\partial x} \\
& +c p^{\nu-2}\left(\frac{\partial p}{\partial x}\right)^{2}+\ldots+d\left(\frac{\partial p}{\partial x}\right)^{\nu}+\ldots \quad\left(p^{\nu}=\left(p^{(1)}\right)^{\nu_{1}} \ldots\left(p^{(\rho)}\right)^{\nu_{\rho}}\right)
\end{aligned}
$$

and correspondingly $v=B\left(x, u, \frac{\partial u}{\partial x}, \ldots ; p\right)$; then by composition with $z=A\left(y, v, \frac{\partial v}{\partial y}, \ldots ; q\right)$, for the terms of lowest order, we get

$$
z=x+\sum a\left(p^{\nu}+q^{\nu}\right)+b\left\{p^{\nu-1} \frac{\partial p}{\partial x}+q^{\nu-1} \frac{\partial q}{\partial x}\right\}+c\left\{p^{\nu-2}\left(\frac{\partial p}{\partial x}\right)^{2}+q^{\nu-2}\left(\frac{\partial q}{\partial x}\right)^{2}\right\}+\ldots
$$

\footnotetext{
${ }^{15} \psi_{i}=0$, or, somewhat more generally, $\psi_{i}=T_{i}$, where $T_{i}$ are newly adjoined functions, are referred to in physics as "field equations." In the case $\psi_{i}=T_{i}$, the identities (13) goes over into equations $\operatorname{Div} B^{(\lambda)}=\sum T_{i} \delta u_{i}^{(\lambda)}$, likewise known in physics as laws of conservation.

${ }^{16}$ Provided $f$ is non-linear in the $\kappa$-th derivatives.

${ }^{17}$ Otherwise we also have $u^{\prime \lambda}=$ const. for every $\lambda$, corresponding to
}

$$
u^{\prime \prime} \cdot\left(u^{\prime}\right)^{\lambda-1}=\frac{1}{\lambda} \frac{d}{d x}\left(u^{\prime}\right)^{\lambda} .
$$


Here, if any coefficient different from $a$ and $b$ is different from zero, in other words, if a term $p^{\nu-\sigma}\left(\frac{\partial p}{\partial x}\right)^{\sigma}+q^{\nu-\sigma}\left(\frac{\partial q}{\partial x}\right)^{\sigma}$ actually occurs for $\sigma>1$ it cannot be written as a differential quotient of a single function or power product of one; the number of arbitrary functions, contrary to hypothesis, has thus increased. If all coefficients different from $a$ and $b$ vanish, then, according to the values of the exponents $\nu_{1}, \ldots, \nu_{\rho}$, the second term will become the differential quotient of the first (as always, for example, for a $\mathfrak{G}_{\infty 1}$ ), so that linearity does actually result; or else the number of arbitrary functions must again increase. The infinitesimal transformations, then, owing to the linearity of the $p(x)$ 's, satisfy a system of linear partial differential equations; and since the group property is satisfied, they constitute an "infinite group of infinitesimal transformations" accord to Lie's definition (Grundlagen, $\S 10$ ).

Now the converse is arrived at similarly to the case of the finite group. The fact that the dependencies (16) hold leads, through multiplication by $p^{(\lambda)}$ and addition, by virtue of the identity transformation (14), to $\sum \psi_{i} \bar{\delta} u_{i}=\operatorname{Div} \Gamma$ and thence, as in Section 3 follows the determination of $\Delta x$ and $\Delta u$ and the invariance of $I$ with respect to these infinitesimal transformations, which do actually depend linearly on $\rho$ arbitrary functions and their derivatives up to the $\sigma$-th order. The fact that these infinitesimal transformations, if they contain no derivatives $\frac{\partial u}{\partial x}, \ldots$, certainly form a group, follows, as in Section 3, from the fact that otherwise, by composition more arbitrary functions would occur, whereas by assumption there are to be only $\rho$ dependencies (16); hence they form an "infinite group of infinitesimal transformations." But such a one consists (Grundlagen, Theorem VII, p. 391) of the most general infinitesimal transformations of a certain "infinite group $\mathfrak{G}$ of finite transformations," in Lie's sense, thereby defined. Each such finite transformation is generated from infinitesimal ones (Grundlagen, $\S 7$ ) 18 and so arises through integration of the simultaneous system

$$
\frac{d x_{i}}{d t}=\Delta x_{i} ; \quad \frac{d u_{i}}{d t}=\Delta u_{i} ; \quad\left(x_{i}=y_{i}, u_{i}=v_{i}, \text { for } t=0\right),
$$

where, however, it may be necessary further to assume the arbitrary $p(x)$ 's dependent on $t$. Thus $\mathfrak{G}$ does actually depend on $\rho$ arbitrary functions; if in particular it suffices to assume $p(x)$ free from $t$, then this dependency becomes analytic in the arbitrary function $q(x)=t \cdot p(x) 19$ If derivatives $\frac{\partial u}{\partial x}$, $\ldots$, occurs, it may be necessary also to adjoin infinitesimal transformation $(\mathrm{s}) \bar{\delta} u=0, \operatorname{Div}(f \cdot \Delta x)=0$ before drawing the same conclusions.

In terms of an example of Lie's (Grundlagen, $\S 7$ ), let us add mention of a fairly general case in which it is possible to break through to explicit formulas, which at the same time show that the derivatives of the arbitrary functions up to the $\sigma$-th order to occur; where, in other words, the converse is complete. I refer to such groups of infinitesimal transformations of the $u$ 's thereby "induced" corresponds; i.e., such transformations of the $u$ 's for which $\Delta u$, and consequently $u$, depend only on the arbitrary functions occurring in $\Delta x$; assuming further that the derivatives $\frac{\partial u}{\partial x}$, $\ldots$ do not occur in $\Delta u$. That is we have

$$
\Delta x_{i}=p^{(i)}(x) ; \quad \Delta u_{i}=\sum_{\lambda=1}^{n}\left\{a^{(\lambda)}(x, u) p^{(\lambda)}+b^{(\lambda)} \frac{\partial p^{(\lambda)}}{\partial x}+\ldots+c^{(\lambda)} \frac{\partial^{\sigma} p^{(\lambda)}}{d x^{\sigma}}\right\} .
$$

Since the infinitesimal transformation $\Delta x=p(x)$ generates every transformation $\Delta x=y+g(y)$ with arbitrary $g(y)$, we can in particular determine $p(x)$ to depend on $t$ in such a matter as to generate the single-member group

$$
x_{i}=y_{i}+t \cdot g_{i}(y),
$$

\footnotetext{
${ }^{18}$ Hence it follows in particular that the group $\mathfrak{G}$ generated from the infinitesimal transformations $\Delta x, \Delta u$ of a $\mathfrak{G}_{\infty \rho}$ reduces back to $\mathfrak{G}_{\infty \rho}$. For $\mathfrak{G}_{\infty \rho}$ contains no infinitesimal transformations distinct from $\Delta x, \Delta u$ dependent on arbitrary functions, and cannot contain any independent of them but depending on parameters, as otherwise it would be a mixed group. But according to the above, the infinitesimal transformations determine the finite ones.

${ }^{19}$ The question whether perhaps this latter case always occurs was raised in a different formulation by Lie (Grundlagen, $\S 7$ and $\S 13$ at end).
} 
which goes over into the identity for $t=0$ and into the required $x=y+g(y)$ for $t=1$. For by differentiation of (18), it follows that

$$
\frac{d x_{i}}{d t}=g_{i}(y)=p^{(i)}(x, t)
$$

where $p(x, t)$ is determined from $g(y)$ by inversion of (18); and conversely, (18) is generated from (19) by virtue of the auxiliary condition $x_{i}=y_{i}$ for $t=0$, by which the integral is uniquely determined. By means of (18), the $x$ 's can be replaced in $\Delta u$ by the "constants of integration" $y$ and by $t$; the $g(y)$ 's occurring just up to the $\sigma$-th derivative, the $\frac{\partial y}{\partial x}$ 's being expressed in terms of $\frac{\partial x}{\partial y}$ in $\frac{\partial p}{\partial x}=\sum \frac{\partial g}{\partial y_{\kappa}} \frac{\partial y_{\kappa}}{\partial x}$, and $\frac{\partial^{\sigma} p}{\partial x^{\sigma}}$ being in general replaced by its value in $\frac{\partial g}{\partial y}, \ldots, \frac{\partial x}{\partial y}, \ldots, \frac{\partial^{\sigma} x}{\partial y^{\sigma}}$. For the determination of the $u$ 's we thus obtain the system of equations

$$
\frac{d u_{i}}{d t}=F_{i}\left(g(y), \frac{\partial g}{\partial y}, \ldots \frac{\partial^{\sigma} g}{\partial y^{\sigma}}, u, t\right) \quad\left(u_{i}=v_{i} \text { for } t=0\right)
$$

in which only $t$ and $u$ are variables, while the $g(y), \ldots$ pertain to the field of coefficients, so that integration yields

$$
u_{i}=v_{i}+B_{i}\left(v, g(y), \frac{\partial g}{\partial y}, \ldots \frac{\partial^{\sigma} g}{\partial y^{\sigma}}, t\right)_{t=1},
$$

or transformations depending on exactly $\sigma$ derivatives of the arbitrary functions. The identity is contained in this, by (18), for $g(y)=0$; and the group property follows from the fact that the method specified affords every transformation $x=y+g(y)$, whereby the induced transformation of the $u$ 's is uniquely determined, and the group $\mathfrak{G}$ accordingly exhausted.

From the converse it follows incidentally that it constitutes no restriction to assume the arbitrary functions to be dependent only on the $x$ 's, not on the $u, \frac{\partial u}{\partial x}, \ldots$ For in the latter event, the identity transformation (14), and hence also (15), would involve not only the $p^{(\lambda)}$ 's but also $\frac{\partial p^{(\lambda)}}{\partial u}$, $\frac{\partial p^{(\lambda)}}{\partial \frac{\partial u}{\partial x}, \ldots}$. Now if we assume the $p^{(\lambda)}$ 's to be successively of the zeroth, first, ... degree in $u, \frac{\partial u}{\partial x}$, ..., with arbitrary functions of $x$ as coefficients, then we again obtain dependencies (16), only in greater number; which, however, according to the above converse, through conjunction with arbitrary functions dependent on $x$ only, reduce to the previous case. In the same way it is shown that mixed groups correspond to simultaneous occurrence of dependencies and of divergence relationships independent of them 20

\footnotetext{
${ }^{20} \mathrm{As}$ in Section 3, it here again follows from the converse that besides $I$, every integral $I^{*}$ different from it by an integral over a divergence likewise admits of an infinite group, with the same $\bar{\delta} u$ 's, though $\Delta x$ and $\Delta u$ will in general involve derivatives of the $u$ 's. Such an integral $I^{*}$ was introduced by Einstein in the general theory of relativity to obtain a simpler version of laws of conservation of energy; I specify the infinitesimal transformations that this $I^{*}$ admits of, adhering precisely in nomenclature to Klein's second Note. The integral $I=\int \ldots \int K d \omega=\int \ldots \int \mathfrak{K} d S$ admits of the group of all transformations of the $\omega$ 's and those induced thereby for the $g_{\mu \nu}$ 's; to this correspond the dependencies (Klein's (30))
}

$$
\sum \mathfrak{K}_{\mu \nu} g_{\tau}^{\mu \nu}+2 \sum \frac{\partial g^{\mu \nu} \mathfrak{K}_{\mu \tau}}{\partial \omega^{\sigma}}=0
$$

Now $I^{*}=\int \ldots \int \mathfrak{K}^{*} d S$, where $\mathfrak{K}^{*}=\mathfrak{K}+$ Div, and consequently $\mathfrak{K}_{\mu \nu}^{*}=\mathfrak{K}_{\mu \nu}$, where $\mathfrak{K}_{\mu \nu}^{*}, \mathfrak{K}_{\mu \nu}$ stand in each instance for the Lagrange expressions. The dependencies specified are therefore such for $\mathfrak{K}_{\mu \nu}^{*}$ also; and after multiplication by $p^{\tau}$ and addition, we obtain, applying the transformations of product differentiation in reverse,

$$
\begin{gathered}
\sum \mathfrak{K}_{\mu \nu} p^{\mu \nu}+2 \operatorname{Div}\left(\sum g^{\mu \sigma} \mathfrak{K}_{\mu \tau} p^{\tau}\right)=0 ; \\
\delta \mathfrak{K}^{*}+\operatorname{Div} \sum\left(2 g^{\mu \sigma} \mathfrak{K}_{\mu \tau} p^{\tau}-\frac{\partial \mathfrak{K}^{*}}{\partial g_{\sigma}^{\mu \nu}} p^{\mu \nu}\right)=0 .
\end{gathered}
$$

Comparing this with Lie's differential equation $\delta \mathfrak{K}^{*}+\operatorname{Div}\left(\mathfrak{K}^{*} \Delta \omega\right)=0$,

$$
\Delta \omega^{\sigma}=\frac{1}{\mathfrak{K}^{*}} \cdot \sum\left(2 g^{\mu \sigma} \mathfrak{K}_{\mu \tau} p^{\tau}-\frac{\partial \mathfrak{K}^{*}}{\partial g_{\sigma}^{\mu \nu}} p^{\mu \nu}\right) ; \quad \Delta g^{\mu \nu}=p^{\mu \nu}+\sum g_{\sigma}^{\mu \nu} \Delta \omega^{\sigma}
$$




\section{$\S 5$. Invariance of the Several Constituents of the Relations}

If we specialize the group $\mathfrak{G}$ to be the simplest case usually considered by allowing no derivatives of the $u$ 's in the transformations, and in that the transformed independent variables depend only on the $x$ 's not on the $u$ 's, we can infer invariance of the several constituents in formulas. To begin with, by known arguments, we get invariance of $\int \ldots \int\left(\sum \psi \delta u_{i}\right) d x$; relative invariance, that is of $\sum \psi_{i} \delta u_{i}{ }^{21}$ meaning by $\delta$ any variation. For we have in the first place

$$
\delta I=\int \ldots \int \delta f\left(x, u, \frac{\partial u}{\partial x}, \ldots\right) d x=\int \ldots \int \delta f\left(y, v, \frac{\partial v}{\partial y}, \ldots\right) d y
$$

and in the second place, for $\delta u, \delta \frac{\partial u}{\partial x}, \ldots$ vanishing at the boundary, according to which $\delta v, \delta \frac{\partial v}{\partial y}, \ldots$ vanishing at the boundary also owing to the linear homogeneous transformation of the $\delta u, \delta \frac{\partial u}{\partial x}, \ldots$,

$$
\begin{aligned}
\int \ldots \int \delta f\left(x, u, \frac{\partial u}{\partial x}, \ldots\right) d x & =\int \ldots \int\left(\sum \psi_{i}(u, \ldots) \delta u_{i}\right) d x \\
\int \ldots \int \delta f\left(y, v, \frac{\partial v}{\partial y}, \ldots\right) d y & =\int \ldots \int\left(\sum \psi_{i}(v, \ldots) \delta v_{i}\right) d y
\end{aligned}
$$

and consequently, for $\delta u, \delta \frac{\partial u}{\partial x}, \ldots$ vanishing at the boundary,

$$
\begin{gathered}
\int \ldots \int\left(\sum \psi_{i}(u, \ldots) \delta u_{i}\right) d x=\int \ldots \int\left(\sum \psi_{i}(v, \ldots) \delta v_{i}\right) d y \\
=\int \ldots \int\left(\sum \psi_{i}(v, \ldots) \delta v_{i}\right)\left|\frac{\partial y_{i}}{\partial x_{\kappa}}\right| d x .
\end{gathered}
$$

If in the third integral $y, v, \delta v$ are expressed in terms of $x, u, \delta u$, and the third is equated to the first, we thus have a relationship

$$
\int \ldots \int\left(\sum \chi_{i}(u, \ldots) \delta u_{i}\right) d x=0
$$

for $\delta u$ vanishing at the boundary but otherwise arbitrary, and thence follows, familiarly, the vanishing of the integrand for any $\delta u$ whatever; the relation

$$
\sum \psi_{i}(u, \ldots) \delta u_{i}=\left|\frac{\partial y_{i}}{\partial x_{\kappa}}\right|\left(\sum \psi_{i}(v, \ldots) \delta v_{i}\right)
$$

identical in $\delta u$, therefore holds, asserting the relative invariance of $\sum \psi_{i} \delta u_{i}$ and consequently the invariance of $\int \ldots \int\left(\sum \psi_{i} \delta u_{i}\right) d x 22$

follow as infinitesimal transformations of which $I^{*}$ admits. These infinitesimal transformations, then depend on the first and second derivatives of the $g^{\mu \nu}$ 's, and contain the arbitrary $p$ 's as far as the first derivative.

${ }^{21}$ That is, $\sum \psi_{i} \delta u_{i}$ takes on a factor upon transformation, and this always used to be termed relative invariance in the algebraic theory of invariance.

${ }^{22}$ These conclusions fail if $y$ depends also on the $u$ 's, since in that case $\delta f\left(y, v, \frac{\partial v}{\partial y}, \ldots\right)$ also contains terms $\sum \frac{\partial f}{\partial y} \delta y$, so that the divergence transformation does not lead to the Lagrange expressions; and similarly if derivatives of the $u$ 's are admitted; for in that case the $\delta v$ 's become linear combinations of $\delta u, \delta \frac{\partial u}{\partial x}, \ldots$, and so lead only after another divergence transformation to an identity $\int \ldots \int\left(\sum \chi_{i}(u, \ldots) \delta u\right) d x=0$, so that again the Lagrange expressions do not appear on the right.

The question whether it is possible to argue from the invariance of $\int \ldots \int\left(\sum \psi_{i} \delta u_{i}\right) d x$ back to the subsistence of divergence relationships is synonymous, according to the converse, with the question whether one can thence infer the invariance of $I$ with respect to a group leading not necessarily to the same $\Delta u, \Delta x$, but to the same $\bar{\delta} u$ 's. In the special case of the single integral and only first derivatives in $f$, it is possible for the finite group to argue from the invariance of the Lagrange expressions to the existence of first integrals (c.f. e.g., Engel, Gött. Nachr. 1916, p. 270). 
To apply this to the divergence relationships and dependencies derived, we must first demonstrate that the $\bar{\delta} u$ derived from the $\Delta u, \Delta x$ 's does in fact satisfy the laws of transformation for the variation $\delta u$, provided only that the parameters, or arbitrary functions, in $\bar{\delta} v$ are so determined as corresponds to the similar group of infinitesimal transformations in $y, v$; if $\mathfrak{T}_{q}$ designates the transformation that carries $x, u$ over into $y, v$, and $\mathfrak{T}_{p}$ and infinitesimal one in $x, u$, then the one similar thereto in $y$, $v$ is given by $\mathfrak{T}_{r}=\mathfrak{T}_{q} \mathfrak{T}_{p} \mathfrak{T}_{q}^{-1}$, where the parameters, or arbitrary functions $r$, are thus determined from $p$ and $q$. In formulas, this is expressed as follows:

$$
\begin{gathered}
\mathfrak{T}_{p}: \xi=x+\Delta x(x, p) ; \quad u^{*}=u+\Delta u(x, u, p) ; \\
\mathfrak{T}_{q}: y=A(x, q) ; \quad v=B(x, u, q) ; \\
\mathfrak{T}_{q} \mathfrak{T}_{p}: \eta=A(x+\Delta x(x, p), q) ; \quad v^{*}=B(x+\Delta x(p), u+\Delta u(p), q) .
\end{gathered}
$$

But this generates $\mathfrak{T}_{r}=\mathfrak{T}_{q} \mathfrak{T}_{p} \mathfrak{T}_{q}^{-1}$, or

$$
\eta=y+\Delta y(r) ; \quad v^{*}=v+\Delta v(r),
$$

if by the inverse $\mathfrak{T}_{q}$ we regard the $x$ 's as functions of the $y$ 's and consider only the infinitesimal terms; so we have the identity

$$
\begin{gathered}
\eta=y+\Delta y(r)=y+\sum \frac{\partial A(x, q)}{\partial x} \Delta x(p) \\
v^{*}=v+\Delta v(r)=v+\sum \frac{\partial B(x, u, q)}{\partial x} \Delta x(p)+\sum \frac{\partial B(x, u, q)}{\partial u} \Delta u(p) .
\end{gathered}
$$

Replacing $\xi=x+\Delta x$ by $\xi-\Delta \xi$ in this so that $\xi$ goes back into $x$, and $\Delta x$ vanishes, by the first equation (20) $\eta$ too will go back over into $y=\eta-\Delta \eta$; if by this substitution $\Delta u(p)$ goes over into $\bar{\delta} u(p)$, then $\Delta v(r)$ will go over into $\bar{\delta} v(r)$ as well, and the second formula (20) gives

$$
\begin{aligned}
v+\bar{\delta} v(y, v, \ldots r) & =v+\sum \frac{\partial B(x, u, q)}{\partial u} \bar{\delta} u(p), \\
\bar{\delta} v(y, v, \ldots r) & =\sum \frac{\partial B}{\partial u_{\kappa}} \bar{\delta} u_{\kappa}(x, u, p),
\end{aligned}
$$

so that the transformation formulas for variations are actually satisfied provided $\bar{\delta} v$ is assumed to depend only on the parameters or arbitrary functions $r 23$

So in particular, the relative invariance of $\sum \psi_{i} \bar{\delta} u_{i}$ follows: hence also, by $(12)$, since the divergence relationships are satisfied in $y, v$ as well, the relative invariance of Div $B$; and further, by (14) and (13), the relative invariance of Div $\Gamma$ and of the left-hand sides of the dependencies as conjoined with the $p^{(\lambda)}$ 's, where the arbitrary $p(x)$ 's (or the parameters) are to be replaced by the $r$ 's everywhere in the transformed formulas. This leads as well to the relative invariance of $\operatorname{Div}(B-\Gamma)$, or of a divergence of a not identically vanishing system of functions $B-\Gamma$ whose divergence vanishes identically.

From the relative invariance of $\operatorname{Div} B$, we can draw additional inference of invariance of the first integral in the one-dimensional case and for finite group. The parametric transformation corresponding to the infinitesimal transformation becomes, by (20), linear and homogeneous, and owing to the invertibility of all transformations, the $\epsilon$ 's also will be linear and homogeneous in the transformed parameters $\epsilon^{*}$. This invertibility is certainly preserved if we put $\psi=0$, since no derivatives of the $u$ 's occur in (20). Through equating the coefficients of the $\epsilon^{*}$ 's in

$$
\operatorname{Div} B(x, u, \ldots \epsilon)=\frac{d y}{d x} \cdot \operatorname{Div} B\left(y, v, \ldots \epsilon^{*}\right)
$$

\footnotetext{
${ }^{23}$ It turns out again that $y$ must be taken independent of $u$ in order for the conclusions to hold. As an example, consider the $\delta g^{\mu \nu}$ and $\delta q_{\rho}$ given by Klein, which satisfy the transformations for variations provided the $p$ 's are subjected to a vector transformation.
} 
the $\frac{d}{d y} B^{(\lambda)}(y, v, \ldots)$ 's therefore also become linear homogeneous functions of $\frac{d}{d x} B^{(\lambda)}(x, u, \ldots)$ 's so that $\frac{d}{d x} B^{(\lambda)}(x, u, \ldots)=0$ or $B^{(\lambda)}(x, u)=$ const. duly entails $\frac{d}{d y} B^{(\lambda)}(y, v, \ldots)=0$ or $B^{(\lambda)}(y, v)=$ const. as well. So the $\rho$ first integrals corresponding to a $\mathfrak{G}_{\rho}$ in each instance admit of the group, with result that the further integration is simplified. The simplest example of this is that $f$ is free of $x$ or of a $u$, which corresponds to the infinitesimal transformation $\Delta x=\epsilon, \Delta u=0$, or $\Delta x=0$, $\Delta u=\epsilon$. We shall have $\bar{\delta} u=-\epsilon \frac{d u}{d x}$ or $\epsilon$ respectively, and since $B$ is derived from $f$ and $\bar{\delta} u$ by differentiation and rational combination, it is free accordingly of $x$ or $u$ respectively, and admits of the corresponding groups 24

\section{§ 6. A Hilbertian Assertion}

From the foregoing, finally, we also obtain the proof of a Hilbertian assertion about the connection of the failure of laws of conservation of energy proper with "general relativity" (Klein's first Note, Göttinger Nachr. 1917, Reply 1st paragraph), and that in a generalized group theory version.

Let the integral $I$ admit of a $\mathfrak{G}_{\infty \rho}$, and let $\mathfrak{G}_{\rho}$ be any finite group generated by specializing the arbitrary functions, and hence a subgroup of $\mathfrak{G}_{\infty}$. Then to the infinite group $\mathfrak{G}_{\infty \rho}$ there correspond dependencies (16), and to the finite one $\mathfrak{G}_{\sigma}$, divergence relationships (13); and conversely from the subsistence of any divergence relationships, the invariance of $I$ follows, with respect to some finite group which will be identical with $\mathfrak{G}_{\sigma}$ if and only if the $\bar{\delta} u$ 's are linear combinations of those obtained from $\mathfrak{G}_{\sigma}$. Thus the invariance with respect to $\mathfrak{G}_{\sigma}$ cannot lead to any divergence relationships different from (13). But since the subsistence of (16) entails the invariance of $I$ with respect to the infinitesimal transformations, $\Delta u, \Delta x$ of $\mathfrak{G}_{\infty \rho}$ for any $p(x)$, it entails in particular nothing less than invariance with respect to the infinitesimal transformations of $\mathfrak{G}_{\sigma}$ arising therefrom by specialization and consequently with respect to $\mathfrak{G}_{\sigma}$. Thus the divergence relationships $\sum \psi_{i} \bar{\delta} u_{i}^{(\lambda)}=\operatorname{Div} B^{(\lambda)}$ must be consequences of the dependencies (16), which latter may alternatively be written $\sum \psi_{i} a_{i}^{(\lambda)}=$ $\operatorname{Div} \chi^{(\lambda)}$ where the $\chi^{(\lambda)}$ 's are linear combinations of the Lagrange expressions and their derivatives. Since the $\psi$ 's occur linearly in both (13) and (16), the divergence relations must thus in particular be linear combinations of the dependencies (16); Accordingly, Div $B^{(\lambda)}=\operatorname{Div}\left(\sum \alpha \cdot \chi^{(\kappa)}\right)$; and the $B^{(\lambda)}$ 's themselves are thus linearly composed of the $\chi$ 's, i.e., the Lagrange expressions and their derivatives, and of functions whose divergence vanishes identically, say like the $B-\Gamma$ 's encountered at the close of Section 2, for which $\operatorname{Div}(B-\Gamma)=0$, and where the divergence at the same time has the invariant property. I shall refer to divergence relationships in which the $B^{(\lambda)}$ 's can be composed from the Lagrange expressions and their derivatives in the specified manner as "improper," and to all other as "proper."

If conversely the divergence relations are linear combinations of the dependencies (16), and so "improper," invariance with respect to $\mathfrak{G}_{\sigma}$ follows from that with respect to $\mathfrak{G}_{\infty \rho} ; \mathfrak{G}_{\sigma}$ becomes a subgroup of $\mathfrak{G}_{\infty \rho}$. The divergence relationships corresponding to an infinite group $\mathfrak{G}_{\sigma}$ will thus be improper if and only if $\mathfrak{G}_{\sigma}$ is a subgroup of an infinite group invariant with respect to $I$.

By specialization of the groups, this yields the original Hilbertian assertion. Let "displacement group" be understood to mean the finite

$$
y_{i}=x_{i}+\epsilon_{i} ; \quad v_{i}(y)=u_{i}(x)
$$

\footnotetext{
${ }^{24}$ In the cases where mere invariance of $\int\left(\sum \psi_{i} \delta u_{i}\right) d x$ entails the existence of first integrals, these do not admit of the entire group $\mathfrak{G}_{\rho}$; for example, $\int\left(u^{\prime \prime} \delta u\right) d x$ admits of the infinitesimal transformation $\Delta x=\epsilon_{2}, \Delta u=\epsilon_{1}+x \epsilon_{3}$; whereas the first integral $u-u^{\prime} x=$ const., corresponding to $\Delta x=0, \Delta u=x \epsilon_{3}$, does not admit of the other two infinitesimal transformations, since it explicitly contains both $u$ and $x$. To this first integral, there happen to correspond infinitesimal transformations for $f$ that contain derivatives. So we see that invariance $\int \ldots \int\left(\sum \psi_{i} \delta u_{i}\right) d x$ is at all events a weaker condition than invariance of $I$, and this should be noted as to a question raised in a previous remark.
} 
that is

$$
\Delta x_{i}=\epsilon_{i}, \quad \Delta u_{i}=0, \quad \bar{\delta} u_{i}=-\sum_{\lambda} \frac{\partial u_{i}}{\partial x_{\lambda}} \epsilon_{\lambda} .
$$

Invariance with respect to the displacement group asserts, as we know, that in $I=\int \ldots \int f\left(x, u, \frac{\partial u}{\partial x}, \ldots\right) d x$, the $x$ 's do not occur explicitly in $f$. The associated $n$ divergence relationships

$$
\sum \psi_{i} \frac{\partial u_{i}}{\partial x_{\lambda}}=\operatorname{Div} B^{(\lambda)} \quad(\lambda=1,2, \ldots n)
$$

will be referred to as "energy relationships," since the laws of conservation" $\operatorname{Div} B^{(\lambda)}=0$ corresponding to the variation problem answer to "laws of conservation of energy," and the $B^{(\lambda)}$ 's to the "energy components." So then we have: If $I$ admits of the displacement group, then the energy relationships become improper if and only if $I$ is invariant with respect to an infinite group containing the displacement group as subgroup 25

An example of such infinite groups is presented by the group of all transformations of the $x$ 's and such of the induced transformations of the $u(x)$ 's in which only derivatives of the arbitrary functions $p(x)$ occur; the displacement group is generated by the specialization $p^{(i)}(x)=\epsilon_{i}$; but it must remain undecided whether this - and the groups generated by change of $I$ by a boundary integral - suffices to give the most general of these groups. Induced transformations of the specified kind arise, say, when the $u$ 's are subjected to the coefficient transformations of a "total differential form," i.e., a form $\sum a d^{\lambda} x_{i}+\sum b d^{\lambda-1} x_{i} d x_{\kappa}+\ldots$ containing higher differentials besides the $d x^{\prime}$ 's; more special induced transformations, in which the $p(x)$ 's occur in first derivative only, are furnished by the coefficient transformations of ordinary differential forms $\sum c d x_{i_{1}} \ldots d x_{i_{\lambda}}$, and only these have ordinarily been considered.

Another group of the specified kind - one which, owing to the occurrence of the logarithmic term, cannot be coefficient transformation — is, say, the following:

$$
\begin{aligned}
& y=x+p(x) ; \quad v_{i}=u_{i}+\ln \left(1+p^{\prime}(x)\right)=u_{i}+\ln \frac{d y}{d x} \\
& \Delta x=p(x) ; \quad \Delta u_{i}=p^{\prime}(x) ; 26 \quad \bar{\delta} u_{i}=p^{\prime}(x)-u_{i}^{\prime} p(x)
\end{aligned}
$$

The dependencies (16) here become

$$
\sum_{i}\left(\psi_{i} u_{i}^{\prime}+\frac{d \psi_{i}}{d x}\right)=0
$$

and the improper energy relationships

$$
\sum\left(\psi_{i} u_{i}^{\prime}+\frac{d\left(\psi_{i}+\text { const. }\right)}{d x}\right)=0 .
$$

A simple invariant integral of the group is

$$
I=\int \frac{e^{-2 u_{1}}}{u_{1}^{\prime}-u_{2}^{\prime}} d x
$$

The most general $I$ is determined by integration of Lie's differential equation (11)

$$
\bar{\delta} f+\frac{d}{d x}(f \cdot \Delta x)=0,
$$

\footnotetext{
${ }^{25}$ The laws of conservation of energy of classical mechanics as well as those of the old "theory of relativity" (where $\sum d x^{2}$ goes over into itself) are "proper," since no infinite groups occur.

${ }^{26}$ From these infinitesimal transformations, the finite ones are calculated backwards by the method given in Section 4 at end.
} 
which by substitution of their values for $\Delta x$ and $\bar{\delta} u$, provided $f$ is assumed to depend on only first derivatives of the $u$ 's, goes over into

$$
\frac{\partial f}{\partial x} p(x)+\left\{\sum \frac{\partial f}{\partial u_{i}}-\frac{\partial f}{\partial u_{i}^{\prime}} u_{i}^{\prime}+f\right\} p^{\prime}(x)+\left\{\sum \frac{\partial f}{\partial u_{i}^{\prime \prime}}\right\} p^{\prime \prime}(x)=0
$$

(identically in $p(x), p^{\prime}(x), p^{\prime \prime}(x)$ ). This system of equations has solutions for as few as two functions $u(x)$ actually containing the derivatives, namely

$$
f=\left(u_{1}^{\prime}-u_{2}^{\prime}\right) \Phi\left(u_{1}-u_{2}, \frac{e^{-u_{1}}}{u_{1}^{\prime}-u_{2}^{\prime}}\right),
$$

where $\Phi$ stands for an arbitrary function of the specified arguments.

Hilbert enunciates his assertion to the effect that the failure of proper laws of conservation of energy is a characteristic feature of the "general theory of relativity." In order for this assertion to hold good literally, therefore, the term "general relativity" should be taken in a broader sense than usual, and extended also to the forgoing groups depending on $n$ arbitrary functions 27

\footnotetext{
${ }^{27}$ This again confirms the correctness of a comment of Klein's that the term "relativity" current in physics is replaceable by "invariance relative to a group." ("Über die geometrishen Grundlagen der Lorentzgruppe," Jhrber. d. Deutsch. Math. Vereinig. 19 (1910), p. 287, reprinted in the Phys. Zeitschrift.)
} 